\title{
The Genetics of IgA Nephropathy: An Overview from China
}

\section{Li Zhu Hong Zhang}

Renal Division, Department of Medicine, Peking University First Hospital, Peking University Institute of Nephrology, Key Laboratory of Renal Disease, Ministry of Health of China, and Key Laboratory of Chronic Kidney Disease Prevention and Treatment (Peking University), Ministry of Education, Beijing, China

\section{Key Words}

IgA nephropathy · Genetics · Linkage analyses · Association analyses

\begin{abstract}
Background: IgA nephropathy ( $\lg \mathrm{AN}$ ) is the most common type of primary glomerulonephritis worldwide. Highly variable data for disease prevalence and reports of familial clustering suggest the involvement of genetic factors in IgAN. As China is an area with a high prevalence of IgAN, Chinese scholars have made a considerable effort to reveal the underlying genetic architecture of IgAN. Summary: In this review, we summarize recent achievements in the genetic studies of $\lg \mathrm{AN}$, focusing mainly on studies undertaken in China. Early association studies followed a population-based design and focused on a single variant or single gene. Subsequently, family-based designs and genetic interactions applied by Chinese scholars revealed an association of variants in MEGSIN and glycosyltransferase genes with IgAN. Recently, genome-wide association studies (GWAS) have been used to identify multiple susceptibility loci for IgAN, and they have, for the most part, been validated in Chinese populations. Key Messages: More efforts should be made to explore the underlying genetic mechanisms of GWAS-identified variants. In future studies in IgAN, the application of a
\end{abstract}

systems genetics approach would be helpful and productive. Facts from East and West: The reported prevalence of $\lg A N$ is higher in Asia than in Europe and North America. However, differences in use of biopsy for the diagnosis of IgAN should be taken into account in analyzing data from both East and West. In Europe, IgAN affects men more frequently than women; this is not the case in Asia. Familial IgAN has been more frequently reported in Europe than in Asia. Within Europe, familial IgAN is more evident in southern than in northern populations. Changes in the pattern of serum IgA1 O-glycosylation is a common finding in IgAN patients in the East and West. SNPs within the gene coding for the enzyme C1GALT1 have been reported in Chinese and European patients. However, there is no evidence for a role of gene polymorphism of the C1GALT1 chaperone cosmc in Europeans. Genetic variants in the HLA gene family have been observed in populations from the East and West. Associations between IgAN and variants of the TAP1/PSMB and DEFA genes were observed in Asian but not in Western patients. Association with the angiotensin-converting enzyme gene was seen only in Asian patients. @ 2015 S. Karger AG, Basel

For an overview of the genetics of IgA nephropathy in Western countries, see Feehally and Barratt, Kidney Diseases 2015, DOI: 10.1159/000381738, www.karger.com/doi/10.1159/000381738.

\section{KARGER 125}

C 2015 S. Karger AG, Basel

2296-9381/15/0011-0027\$39.50/0

E-Mail karger@karger.com

www.karger.com/kdd
Hong Zhang, MD, Ph.D.

Renal Division, Department of Medicine, Peking University First Hospital

Peking University Institute of Nephrology

No.8 Xi Shi Ku Street, Beijing 100034 (China)

E-Mail hongzh@bjmu.edu.cn 


\section{Introduction}

IgA nephropathy (IgAN) is the most common type of primary glomerulonephritis worldwide [1]. In China, it accounts for $37-58 \%$ of biopsy-proven primary glomerulonephritis [2-4]. Patients with IgAN bear a broad spectrum of clinical and pathologic changes, and $30 \%$ of them progress to end-stage renal disease within 10 years after the diagnosis $[5,6]$. The high prevalence and chronic progression of IgAN result in a considerable disease burden.

The exact pathogenesis of IgAN is unknown. However, increasing evidence suggests the involvement of genetic factors in the development and progression of IgAN [7]. Genetic studies have led to considerable improvement in knowledge of the pathogenesis of IgAN [8].

As China is an area with a high prevalence of IgAN, Chinese scholars have made considerable efforts to reveal the underlying genetic architecture of IgAN. In this review, after a brief description of the genetic characteristics of IgAN, we summarize recent achievements in the genetic studies of IgAN, focusing mainly on studies undertaken in China.

\section{Genetic Characteristics of IgAN}

The prevalence of IgAN shows considerable differences among geographic and racial populations. The Asian population has a prevalence of $30-60 \%[2-4,9,10]$, the European population one of $20-30 \%$ [11, 12], and the North American population one of $10 \%$ [13]. The influence of polices regarding renal biopsy on the reported prevalence of IgAN should be considered, but genetic factors also contribute to this difference: indigenous North Americans have a IgAN prevalence of 38\% [14], whereas that of African-Americans is $2 \%$ [15].

Moreover, after the first report of two affected siblings with IgAN by Tolkoff-Rubin et al. in 1978 [16], studies about the familial clustering of IgAN from all over the world were published [17-19]. In a study from Italy, patients with IgAN were classified into 'sporadic' (only 1 family member was affected), 'suspected familial' (1 member had biopsy-proven IgAN and others showed persistent microscopic hematuria and refused renal biopsy) and 'familial' (at least 2 individuals had biopsyproven IgAN) [20]. The investigators observed that $>50 \%$ of IgAN patients had familial IgAN or suspected familial IgAN [20]. However, it has been reported that the proportion of individuals in China with familial IgAN and suspected familial IgAN is 1.3 and $7.4 \%$, respectively, which seems to be much lower than that in European subjects. Reports of these pedigrees suggest the genetic disease features of IgAN. However, the striking differences in the prevalence of sporadic IgAN and familial IgAN around the world also imply a role for genetic factors in IgAN.

\section{Genetic Studies of IgAN}

Thanks to the Human Genome Project, hundreds and thousands of genetic markers that span the entire human genome have been discovered. Based on these markers, genetic studies have been conducted widely in recent years. Linkage analyses and genetic association studies for IgAN have been carried out. Linkage studies are based on the theory of genetic linkage and can thus be conducted in genetic pedigrees. The logarithm of the odds score is used in linkage analyses as a statistical test to evaluate the probability that a gene important for a disease is linked to a genetic marker. However, recruiting subjects with the familial forms of IgAN is quite difficult. Therefore, along with the rapid development of genotyping technology, genetic association studies designed for patients with familial and sporadic forms of IgAN have been conducted widely.

\section{Linkage Analyses}

Linkage analyses in IgAN have been reported from three research teams [21-23]. As early as 2000, Gharavi et al. [21] demonstrated linkage of IgAN to 6q22-23 under a dominant model of transmission with incomplete penetrance, and this locus was termed 'IGAN1'. Later, scholars from Europe reported two additional loci linked to IgAN: 4q26-31 (IGAN2) and 17q12-22 (IGAN3) [22]. Another locus identified from a large IgAN pedigree in Canada was located at $2 \mathrm{q} 36$ [23], which is a region that also contains COL4A3 and COL4A4, the causal genes for thin basement membrane disease (TBMD). Patients with IgAN and TBMD share many clinical and pathologic features [24], so whether $2 \mathrm{q} 36$ is linked to IgAN or just to TBMD is unknown [25].

After identifying susceptible loci linked to IgAN, scholars have made considerable efforts to explore the causative genes in those regions, but unfortunately the causative genes have yet to be identified. Many reasons have been postulated regarding the failure of identification of the causative genes of IgAN by linkage analyses. The diagnosis of IgAN is entirely dependent on renal biopsy. Hence, the accurate identification of family mem- 
bers as being 'affected' or 'unaffected' is difficult because of the lack of a reliable noninvasive test for screening. Moreover, the broadly variable clinical presentations of IgAN suggest that this disease may encompass multiple subsets, and that these subsets might result in genetic heterogeneity in patients, even in the familial form. Karnib et al. [26] found no evidence of linkage to the reported IgAN loci on chromosomes 6q22-23, 2q36 and $4 \mathrm{q} 22-31$ in a large Lebanese family with IgAN (five generations and 16 affected individuals). Those findings suggested genetic heterogeneity and called for distinction based on genetic/biomarker data before linkage analyses. In addition, there is uncertainty regarding the genetic model for IgAN. A dominant model with incomplete penetrance has been proposed [21], but the complexity of IgAN suggests that it is a multi-factorial and polygenic disease involving environmental and genetic factors.

\section{Association Studies}

Compared with linkage analyses, association studies have been conducted more widely in China. In the early days, association studies had a population-based design and focused on a single variant or single gene. Then, when association studies were closely associated with a low rate of replication, family-based designs were introduced. With the rapid progress of bioinformatic technology, subsequent association studies have paid more attention to multiple variants in a single gene (haplotype) and genetic interactions in multiple genes. In recent years, rapid improvements in single nucleotide polymorphism (SNP) genotyping technology have led to genome-wide association studies (GWAS). Two GWAS in IgAN based on Chinese populations have been conducted [27, 28]. Multiple loci identified by GWAS are now awaiting functional studies to reveal the underlying genetic pathogenesis.

\section{Early Genetic Association Studies}

Human leukocyte antigen (HLA) gene family [18, 29, 30], renin-angiotensin system-related [31-37], TRAC $[38,39]$ and cytokine-related genes (interleukin-1, transforming growth factor- $\beta 1$ ) [40, 41] have undergone association studies to test the development and/or progression of IgAN.

Reports from Hong Kong, Taiwan and Sichuan have revealed genetic variants in the HLA gene family to be associated with the genetic susceptibility of IgAN, or a special subgroup of IgAN [18, 29, 30]. Renin-angiotensin system-related genes have been postulated as candidate genes for association studies in IgAN but

The Genetics of IgA Nephropathy in China have yielded contradictory results [31-37]. Among variants in the renin-angiotensin system, insertion/deletion (I/D) polymorphisms in angiotensin-converting enzyme (ACE) genes have been observed. Systematic reviews and meta-analyses have demonstrated an association between I/D polymorphisms in ACE genes and IgAN in Asian populations, but genetic association was not so important in Caucasian populations, which might explain the controversial results in previous genetic association studies [31, 33, 34]. Actually, most early genetic association studies based on a candidate-gene approach have not been replicated. Besides genetic heterogeneitybetween ethnic groups, methodological problems (e.g., small sample size, poorly matched controls, inadequate coverage of variants in candidate genes) and a lack of statistical power have made genetic association studies for IgAN using a candidate-gene approach rather unrevealing.

\section{Family-Based Association Studies}

When hundreds of candidate gene-based association studies for IgAN were conducted, many positive genetic associations were noted, but, simultaneously, the number of replications of these associations was low. This phenomenon arose from an intrinsic disadvantage of candidate-based association studies, population stratification, in which mismatched populations between cases and controls elicit spurious associations that could not be validated.

Family-based association studies can be applied to overcome the weakness of population stratification. Unlike a traditional population-based design, the simplest study design for a family-based association, called a 'transmission disequilibrium test' (TDT), uses genotype data from trios. Then, through comparison of the observed number of alleles transmitted with those expected in Mendelian transmissions, the excess of alleles transmitted to affected individuals can be identified, which should be the disease-susceptible alleles. Therefore, a TDT design is not sensitive to population structures, and significant findings suggest linkage and association.

Using a TDT design, Li et al. [42] revealed that MEGSIN C2093T and C2180T confer susceptibility to IgAN. In subsequent studies, involvement of genetic variations of MEGSIN in IgAN progression was demonstrated in Chinese populations $[43,44]$. A recent meta-analysis on seven reports focusing on genetic variations in MEGSIN showed a genetic association between MEGSIN variants and risk of IgAN in an Asian population [45]. 
Recent Association Studies Focusing on Genetic Interactions

In the last decade, a key breakthrough in the study of the pathogenesis of IgAN has been the identification of an abnormality in O-glycosylation in IgA1 molecules [46]. Several studies on pathogenic mechanisms have reported that defects in IgA1 glycosylation lead to the formation of immune complexes and initiate the development of IgAN. More recently, research teams from the USA and China have independently reported that defects in IgA1 glycosylation are an inherited phenotype in patients with familial and sporadic IgAN and their relatives $[47,48]$ and constitute a heritable risk factor for IgAN.

The O-glycan of IgA1 molecules is synthesized by a series of glycosyltransferases. Thus, we conducted genetic association studies with four key glycosyltransferase genes for IgA1 O-glycosylation (C1GALT1, ST6GALNAC2, C1GALT1C1, MUC20) as candidates, and tested the genetic association of variants in these genes in a large population of Chinese subjects with IgAN [49-53]. Our study identified one protective (YATIG) and two risky (YAGDA, YATDG) regulatory haplotypes in the C1GALT1 gene and proposed a genetic association between the C1GALT1 gene and IgAN [49], which was validated independently in three subsequent studies in European and Chinese populations [54-56]. Besides C1GALT1, we also demonstrated that the ADG haplotype in the promoter region of ST6GALNAC2 is a functional regulatory polymorphism that results in a predisposition to IgAN [50]. Furthermore, using two independent multiple-locus analysis algorithms, we demonstrated the genetic interaction between C1GALT1 and ST6GALNAC2 and IgA1 glycosylation, as well as the predisposition and severity of IgAN [52]. This observation suggested that variants in C1GALT1 and ST6GALNAC2, through their influence on IgA1 O-glycosylation, contribute to IgAN in a polygenic manner [52].

\section{Genome-Wide Association Studies}

Four GWAS have been conducted in IgAN populations $[27,28,57,58]$. The first GWAS in IgAN detected about 300,000 SNPs in a European population [57]. In that study, when tested against a significance threshold in GWAS, HLA regions (across loci for HLA-B, DRB1, DQA, and DQB) showed significant associations with IgAN, which was in agreement with the results in earlier genetic association studies, in which a HLA gene family was identified.

The next GWAS used >2,000 Northern Chinese subjects as a discovery cohort and was validated in a Southern Chinese and a Caucasian population [27]. In that study, five associated loci were identified. Besides three independent loci in HLA regions, 1q32 (encompasses CFHCFHRs) and 22q12 (encompasses OSM and LIF) attracted much attention. Based on novel identification by GWAS, our research team further explored the underlying mechanism of genes in 1q32 with IgAN susceptibility [59]. We found that genetic variants in $\mathrm{CFH}, \mathrm{CFHR} 3$ and CFHR1 affected complement activation through their influence on CFH levels and thereby predisposed individuals to develop IgAN. A subsequent GWAS by a Chinese research team focused entirely on a Chinese population [28]. In that study, a Southern Chinese population was used as a discovery cohort, and identified loci were validated further in Northern and Southern Chinese populations. In addition to previous identification, two additional loci were reported: 17p13 (containing TNFSF13) and 8p23 (containing DEFA). Two subsequent functional studies focused on the DEFA gene. Xu et al. [60] revealed that polymorphisms within DEFA genes are involved in gene transcriptional regulation, and Qi et al. [61] reported the association of polymorphisms in the DEFA gene with the clinical phenotype of gross hematuria, which implied the role of mucosal immunity in pathogenesis of IgAN. The most recent GWAS in IgAN were carried out in 20,612 individuals of European and East Asian ancestry [58]. The enlarged population provided increased power for the identification of new loci. Actually, six new genome-wide significant associations, four in ITGAM-ITGAX, VAV3 and CARD9 and two new independent signals at HLA-DQB1 and DEFA were reported. When evaluating the association with pathogen diversity and upon application of a pathway-based GWAS approach to the identified IgAN-susceptible variants, a possible role for interactions between hosts and intestinal pathogens were suggested to shape the genetic landscape of IgAN. However, the exact functions of these newly identified loci have yet to be revealed.

\section{Future Perspectives on Genetic Studies in IgAN}

Chinese scholars have participated actively in genetic studies of IgAN, especially in recent GWAS. Cumulatively, we have identified (by GWAS) 15 loci for IgAN susceptibility, but the underlying genetic mechanism is unknown. There is an urgent need to understand how these genetic variants contribute to disease susceptibility. Therefore, in the search for novel IgAN-susceptible loci, more efforts should be made to explore the underlying function of the associated genetic variants. Typically,
30

Kidney Dis 2015;1:27-32 DOI: $10.1159 / 000381740$
Zhu/Zhang 
most of the loci identified by GWAS for common diseases show mild-to-modest effects. Hence, definition of the mechanistic effects of genetic variants on clinical traits using classical molecular biology approaches will be difficult. The application of a systems genetics approach, which uses a range of experimental and statistical methods to integrate multiple levels of information (transcription as well as protein and metabolite levels) [62], would be a helpful and productive strategy in future genetic studies in IgAN.

\section{Acknowledgement}

This work was supported by grants from the Major State Basic Research Development Program of China (program 973, No. 2012CB517700); the Natural Science Foundation for Innovation Research Group of China (81321064); the Capital of Clinical Characteristics and the Applied Research Fund (Z141107002514037); the National Science Foundation of China (grant No. 81470945) and the Beijing Natural Science Foundation (grant No. 7131016).

\section{Disclosure Statement}

The authors have no competing financial interests to declare.

\section{References}

1 D'Amico G: The commonest glomerulonephritis in the world: IgA nephropathy. Q J Med 1987;64:709-727.

2 Zhou FD, Zhao MH, Zou WZ, et al: The changing spectrum of primary glomerular diseases within 15 years: a survey of 3331 patients in a single Chinese centre. Nephrol Dial Transplant 2009;24:870-876.

3 Li LS, Liu ZH: Epidemiologic data of renal diseases from a single unit in China: analysis based on 13,519 renal biopsies. Kidney Int 2004;66:920-923.

-4 Pan X, Xu J, Ren H, et al: Changing spectrum of biopsy-proven primary glomerular diseases over the past 15 years: a single-center study in China. Contrib Nephrol 2013;181:22-30.

5 Barratt J, Feehally J: IgA nephropathy. J Am Soc Nephrol 2005;16:2088-2097.

6 D'Amico G. Natural history of idiopathic IgA nephropathy and factors predictive of disease outcome. Semin Nephrol 2004;24:179-196.

7 Kiryluk K, Novak J: The genetics and immunobiology of IgA nephropathy. J Clin Invest 2014;124:2325-2332.

8 Kiryluk K, Novak J, Gharavi AG: Pathogenesis of immunoglobulin A nephropathy: recent insight from genetic studies. Annu Rev Med 2013;64:339-356.

$\checkmark 9$ Chang JH, Kim DK, Kim HW, et al: Changing prevalence of glomerular diseases in Korean adults: a review of 20 years of experience. Nephrol Dial Transplant 2009;24:2406-2410.

10 Kitajima T, Murakami M, Sakai O: Clinicopathological features in the Japanese patients with IgA nephropathy. Jpn J Med 1983;22: 219-222.

11 Stratta P, Segoloni GP, Canavese C, et al: Incidence of biopsy-proven primary glomerulonephritis in an Italian province. Am J Kidney Dis 1996;27:631-639.

12 Simon P, Ramee MP, Boulahrouz R, et al: Epidemiologic data of primary glomerular diseases in western France. Kidney Int 2004;66: 905-908.
13 Wyatt RJ, Julian BA, Baehler RW, et al: Epidemiology of IgA nephropathy in central and eastern Kentucky for the period 1975 through 1994. Central Kentucky Region of the Southeastern United States IgA Nephropathy DATABANK Project. J Am Soc Nephrol 1998;9: 853-858.

14 Smith SM, Tung KS: Incidence of IgA-related nephritides in American Indians in New Mexico. Hum Pathol 1985;16:181-184.

15 Pontier PJ, Patel TG: Racial differences in the prevalence and presentation of glomerular disease in adults. Clin Nephrol 1994;42:7984.

16 Tolkoff-Rubin NE, Cosimi AB, Fuller T, et al: IGA nephropathy in HLA-identical siblings. Transplantation 1978;26:430-433.

17 Masuda J, Shiiki H, Fujii Y, et al: Identical twin sisters with IgA nephropathy (in Japanese). Nippon Jinzo Gakkai Shi 1996;38:5256.

18 Li PK, Burns AP, So AK, et al: Familial IgA nephropathy: a study of HLA class II allogenotypes in a Chinese kindred. Am J Kidney Dis 1992;20:458-462.

19 Julian BA, Quiggins PA, Thompson JS, et al: Familial IgA nephropathy. Evidence of an inherited mechanism of disease. N Engl J Med 1985;312:202-208.

20 Schena FP, Cerullo G, Rossini M, et al. Increased risk of end-stage renal disease in familial IgA nephropathy. J Am Soc Nephrol 2002;13:453-460.

21 Gharavi AG, Yan Y, Scolari F, et al: IgA nephropathy, the most common cause of glomerulonephritis, is linked to $6 \mathrm{q} 22-23$. Nat Genet 2000;26:354-357.

22 Bisceglia L, Cerullo G, Forabosco P, et al: Genetic heterogeneity in Italian families with IgA nephropathy: suggestive linkage for two novel IgA nephropathy loci. Am J Hum Genet 2006;79:1130-1134.
23 Paterson AD, Liu XQ, Wang K, et al: Genome-wide linkage scan of a large family with IgA nephropathy localizes a novel susceptibility locus to chromosome 2q36. J Am Soc Nephrol 2007;18:2408-2415.

24 Packham DK: IgA glomerulonephritis: can they be distinguished without renal biopsy? Nephrology (Carlton) 2007;12:481-486.

25 Kiryluk K, Julian BA, Wyatt RJ, et al: Genetic studies of IgA nephropathy: past, present, and future. Pediatr Nephrol 2010;25:2257-2268.

26 Karnib HH, Sanna-Cherchi S, Zalloua PA, et al: Characterization of a large Lebanese family segregating IgA nephropathy. Nephrol Dial Transplant 2007;22:772-777.

27 Gharavi AG, Kiryluk K, Choi M, et al: Genome-wide association study identifies susceptibility loci for IgA nephropathy. Nat Genet 2011;43:321-327.

28 Yu XQ, Li M, Zhang H, et al: A genome-wide association study in Han Chinese identifies multiple susceptibility loci for IgA nephropathy. Nat Genet 2012;44:178-182.

29 Huang CC, Hu SA, Lin JL, et al: HLA and Chinese IgA nephropathy in Taiwan. Tissue Antigens 1989;33:45-47.

30 Jiyun Y, Guisen L, Li Z, et al: The genetic variants at the HLA-DRB1 gene are associated with primary IgA nephropathy in Han Chinese. BMC Med Genet 2012;13:33.

31 Yong D, Qing WQ, Hua L, et al: Association of angiotensin I-converting enzyme gene insertion/deletion polymorphism and IgA nephropathy: a meta-analysis. Am J Nephrol 2006;26:511-518.

32 Huang HD, Lin FJ, Li XJ, et al: Genetic polymorphisms of the renin-angiotensin-aldosterone system in Chinese patients with endstage renal disease secondary to IgA nephropathy. Chin Med J (Engl) 2010;123:3238-3242.

33 Qin YH, Zhou TB, Su LN, et al: Association between ACE polymorphism and risk of IgA nephropathy: a meta-analysis. J Renin Angiotensin Aldosterone Syst 2011;12:215-223. 
34 Zhou TB, Yin SS, Liang R: A meta-analysis of the association between angiotensin-converting enzyme insertion/deletion gene polymorphism and end-stage renal disease risk in IgA nephropathy patients. J Renin Angiotensin Aldosterone Syst 2013;14:235-241.

>35 Zhu X, Kong D, Zhang L, et al: Correlation analysis of angiotensin-converting enzyme, angiotensinogen, and endothelial nitric oxide synthase gene polymorphisms and the progression of immunoglobulin A nephropathy/ membranous nephropathy. Hum Pathol 2013:44:2806-2813.

-36 Mao S, Huang S: Association of angiotensinogen gene M235T polymorphism with the risk of IgA nephropathy: a meta-analysis. Ren Fail 2014;36:466-472.

-37 Zhong W, Huang Z, Wu Y, et al: Association of aldosterone synthase (CYP11B2) gene polymorphism with IgA nephropathy risk and progression of IgA nephropathy. J Renin Angiotensin Aldosterone Syst 2014, Epub ahead of print.

-38 Li R, Xue C, Li C, et al: TRAC variants associate with IgA nephropathy. J Am Soc Nephrol 2009;20:1359-1367.

39 Lu C, Zhu K, Zhao H, et al: Correlation between TCRCalpha $-560 \mathrm{C} / \mathrm{T}$ polymorphism and the clinical presentation of Uygur IgA nephropathy patients in XinJiang. Asian Pac J Allergy Immunol 2011;29:236-239.

40 Liu ZH, Cheng ZH, Yu YS, et al: Interleukin-1 receptor antagonist allele: is it a genetic link between Henoch-Schönlein nephritis and IgA nephropathy? Kidney Int 1997;51:19381942.

-41 Wang W, Sun Y, Fu Y, et al: The effects of both single-locus and multi-locus interaction on the clinical manifestations of IgA nephropathy in Southern Han Chinese. Nephrol Dial Transplant 2014;29:550-555.

42 Li YJ, Du Y, Li CX, et al: Family-based association study showing that immunoglobulin A nephropathy is associated with the polymorphisms $2093 \mathrm{C}$ and $2180 \mathrm{~T}$ in the $3^{\prime}$ untranslated region of the Megsin gene. J Am Soc Nephrol 2004;15:1739-1743.
43 Xia Y, Li Y, Du Y, et al: Association of MEGSIN 2093C-2180T haplotype at the $3^{\prime}$ untranslated region with disease severity and progression of IgA nephropathy. Nephrol Dial Transplant 2006;21:1570-1574.

44 Xia YF, Huang S, Li X, et al: A family-based association study of megsin A23167G polymorphism with susceptibility and progression of IgA nephropathy in a Chinese population. Clin Nephrol 2006;65:153-159.

45 Zhou TB, Mo XN, Kou QY, et al: Association of Megsin gene polymorphism with IgA nephropathy risk. J Recept Signal Transduct Res 2015;35:122-126.

46 Novak J, Julian BA, Mestecky J, et al: Glycosylation of IgA1 and pathogenesis of IgA nephropathy. Semin Immunopathol 2012;34: 365-382.

47 Lin X, Ding J, Zhu L, et al: Aberrant galactosylation of IgA1 is involved in the genetic susceptibility of Chinese patients with IgA nephropathy. Nephrol Dial Transplant 2009;24: 3372-3375.

48 Gharavi AG, Moldoveanu Z, Wyatt RJ, et al: Aberrant IgA1 glycosylation is inherited in familial and sporadic IgA nephropathy. J Am Soc Nephrol 2008;19:1008-1014.

49 Li GS, Zhang H, Lv JC, et al: Variants of C1GALT1 gene are associated with the genetic susceptibility to IgA nephropathy. Kidney Int 2007;71:448-453.

50 Li GS, Zhu L, Zhang H, et al: Variants of the ST6GALNAC2 promoter influence transcriptional activity and contribute to genetic susceptibility to IgA nephropathy. Hum Mutat 2007;28:950-957.

51 Li GS, Nie GJ, Zhang H, et al: Do the mutations of C1GALT1C1 gene play important roles in the genetic susceptibility to Chinese IgA nephropathy? BMC Med Genet 2009;10: 101.

52 Zhu L, Tang W, Li G, et al: Interaction between variants of two glycosyltransferase genes in IgA nephropathy. Kidney Int 2009; 76:190-198.
53 Li G, Zhang H, Lv J, et al: Tandem repeats polymorphism of MUC20 is an independent factor for the progression of immunoglobulin A nephropathy. Am J Nephrol 2006;26:4349.

54 Bertinetto FE, Calafell F, Roggero S, et al: Search for genetic association between IgA nephropathy and candidate genes selected by function or by gene mapping at loci IGAN2 and IGAN3. Nephrol Dial Transplant 2012; 27:2328-2337.

55 Pirulli D, Crovella S, Ulivi S, et al: Genetic variant of C1GalT1 contributes to the susceptibility to IgA nephropathy. J Nephrol 2009; 22:152-159.

56 Wang W, Sun Y, Fu Y, et al: Interaction of C1GALT1-IL5RA on the susceptibility to IgA nephropathy in Southern Han Chinese. J Hum Genet 2013;58:40-46.

57 Feehally J, Farrall M, Boland A, et al: HLA has strongest association with IgA nephropathy in genome-wide analysis. J Am Soc Nephrol 2010;21:1791-1797.

58 Kiryluk K, Li Y, Scolari F, et al: Discovery of new risk loci for IgA nephropathy implicates genes involved in immunity against intestinal pathogens. Nat Genet 2014;46:1187-1196.

59 Zhu L, Zhai YL, Wang FM, et al: Variants in complement factor $\mathrm{H}$ and complement factor $\mathrm{H}$-related protein genes, CFHR3 and CFHR1, affect complement activation in IgA nephropathy. J Am Soc Nephrol 2014, Epub ahead of print.

60 Xu R, Feng S, Li Z, et al: Polymorphism of DEFA in Chinese Han population with IgA nephropathy. Hum Genet 2014;133:1299_ 1309.

61 Qi YY, Zhou XJ, Cheng FJ, Hou P, Zhu L, Shi SF, Liu LJ, Lv JC, Zhang H: DEFA gene variants associated with IgA nephropathy in a Chinese population. Genes Immun 2015, Epub ahead of print.

62 Civelek M, Lusis AJ: Systems genetics approaches to understand complex traits. Nat Rev Genet 2014;15:34-48. 